\title{
SLEEP DISTURBANCES AND FRAILTY STATUS IN OLDER ADULTS IN VIETNAM
}

\author{
Nguyen Trung Anh ${ }^{1,2}$, Nguyen Thi Ngoc Anh², Dang Thi Xuan ${ }^{3}$ và Nguyen Xuan Thanh ${ }^{1,2,}$, \\ ${ }^{1}$ National Geriatric Hospital \\ ${ }^{2}$ Hanoi Medical University \\ ${ }^{3}$ Vietnam Poison Control Center
}

This cross-sectional study aimed to describe sleep disturbance and its association with frailty syndrome among 903 older adults at the National Geriatric Hospital in Vietnam. Frailty was diagnosed according to the Fried criteria. Sleep disturbances was assessed based on the Pittsburgh Sleep Quality Index (PSQI). Of 903 patients, the mean age was $71.8(S D=8.5)$, and $537(59.5 \%)$ were female. Most (96.7\%) of the patients diagnosed with frailty had poor sleep; $95.3 \%$ had to get up at midnight or early morning; $93.3 \%$ could not sleep within 30 minutes; $86 \%$ had sleep efficiency less than $85 \%$; $53.3 \%$ coughed at night; $50.7 \%$ had nightmares; and $27.3 \%$ and $22.7 \%$ of patients felt hot and cold, respectively. The majority (80.0\%) of patients must get up to use the bathroom. In conclusion, most older adult diagnosed with frailty experienced at least one form of of sleep disturbance. Medical staffs should attend to the patient's sleep quality, especially in patients with frailty.

Keywords: Sleep disturbance, older adults, frailty

\section{INTRODUCTION}

Frailty is a public health problem with a prevalence of about $10.7 \%$ in the communitydwelling elderly population. ${ }^{1}$ In Vietnam, among the hospitalized elderly, the prevalence of pre-frailty and frailty are $39.8 \%$ and $33.8 \%$, respectively. ${ }^{1}$ Frailty older adults are at increased risk of premature death and various negative health outcomes, including falls, fractures, disability, and dementia, all of which could result in poor quality of life, increased cost and use of health care resources, such as emergency department visits, hospitalization, and institutionalization. ${ }^{2-4}$

Sleep disturbances are common and

Corresponding author: Nguyen Trung Anh,

Hanoi Medical University

Email: trunganhvlk@gmail.com

Received: 15/03/2021

Accepted: 20/05/2021 serious problems of the elderly population. ${ }^{5,6} \mathrm{~A}$ 2017 study reported that $28.2 \%$ of Vietnamese elderly have sleep quality disorder. ${ }^{7}$ Sleep is extremely important to health since the human body carries out a series of necessary biological and physiological activities during the sleep process, such as hormonal release, energy metabolism, glucose and cardiovascular regulation, and self-regulation and recovery of physiological functions. $^{8}$

In the world, many studies about sleep disturbances in frailty patients had been conducted and indicated that sleep disturbance was associated with both prevalence and incidentce of frailty among elders. Prior studies showed that the prevalence of sleep disturbances among elders with frailty was $61 \%$ and increased with age, smoking, alcohol intake and some medications. Recently, poor sleep quality and sleep duration were reported 
to be associated with prevalence and incidence of frailty.

Understanding about prevalence of sleep disturbances in frailty patients is highly important managing sleep disturbances and reducing its effect on hospitalization. However, there is so difficulty to find a significant research in Vietnam about this problem. Therefore, we performed this research to describe sleep disturbance and its association with frailty syndrome among older adults.

\section{METHODS}

\section{Research subjects}

903 patients aged 60 and older from the National Geriatric Hospital in Vietnam.

The inclusion criteria were: (1) 60 years old or older and (2) conscious and have the ability to communicate and perform physical tests. Patients who refused to participate or were diagnosed with acute diseases such as stroke, meningitis, encephalorrhagia, etc. were excluded from this study.

\section{Method}

Study design: This was a cross-sectional study

Variables and Data Processing: Participants were interviewed for 20 - 30 minutes. A questionnaire was used to collect demographic data, including age, gender (male or female), educational level (illiterate, primary school, secondary school or higher education), marital status (married, single, widowed, or divorced), and living area (rural area or urban area).

Frailty was defined by Fried's frailty criteria as having 3 or more of the following 5 crieria $^{9}$ : (1) Shrinking; (2) Weakness; (3) Poor endurance;
(4) Slowness; (5) Low physical activity level.

The Pittsburgh Sleep Quality Index (PSQI) was used to assess sleep disturbance. The PSQI score was calculated by summing the points of seven components: (1) Subjective sleep quality; (2) Sleep latency; (3) Sleep duration; (4) Sleep efficiency; (5) Sleep disturbance; (6) Use of sleep mediation; and (7) Daytime dysfunction. If a participant had a PSQI score of $\geq 5$, that participant was considered to have poor sleep.

\section{Data analysis}

Data were coded and entered into REDCap software. Analysis was conducted using SPSS version 22 software. Descriptive statistics were reported as number, percentage, mean and SD. Statistical significance was presented with $p$-value which is less than 0.05 .

\section{Ethics}

All data collected was used for research to improve quality of senior health care service.

\section{RESULTS}

\section{Characteristics of the participants}

Of the 903 participants , $59.5 \%$ (537/903) were female and $40.5 \%$ (366/903) were male; the ratio of female/male was 1.47 . The mean age of the participants was 71.8 years old $(S D=$ 8.5; range: $60-98$ ). The percentage of married participants was $76.5 \%$, which was triple the percentage of unmarried, divorced or widowed participants (23.5\%). About 57.4\% (519) had secondary school and below education, $24.4 \%$ (220) graduated from high school and 18.2\% (164) continued education beyond high school. Only 7 participants $(4.7 \%)$ with frailty had education beyond high school. 
Table 1. Patients Demographics $(n=903)$

\begin{tabular}{llll}
\hline \multicolumn{2}{c}{ Characteristics } & \multicolumn{1}{c}{ N } & \% \\
\hline \multirow{2}{*}{ Age (Mean \pm SD) } & & \multicolumn{2}{c}{$71.8 \pm 8.5$} \\
\hline \multirow{2}{*}{ Gender } & Male & $566(40.5)$ & 40.5 \\
\cline { 2 - 4 } Marital status (missing 3) & Female & $537(59.5)$ & 59.5 \\
\hline \multirow{2}{*}{ Living area (missing 10) } & Married & $689(76.5)$ & 76.5 \\
\cline { 2 - 4 } & Single/Widowed/ Divorced & $211(23.5)$ & 23.5 \\
\hline \multirow{2}{*}{ Educational level (missing 2) } & Rural living & $403(45.1)$ & 45.1 \\
\cline { 2 - 4 } & Urban living & $491(54.9)$ & 54.9 \\
\cline { 2 - 4 } & Secondary school and below & $519(57.4)$ & 57.4 \\
\cline { 2 - 4 } & College/university and above & $164(18.2)$ & 18.2 \\
\hline
\end{tabular}

\section{Prevalence of sleep disturbance in frailty} patients

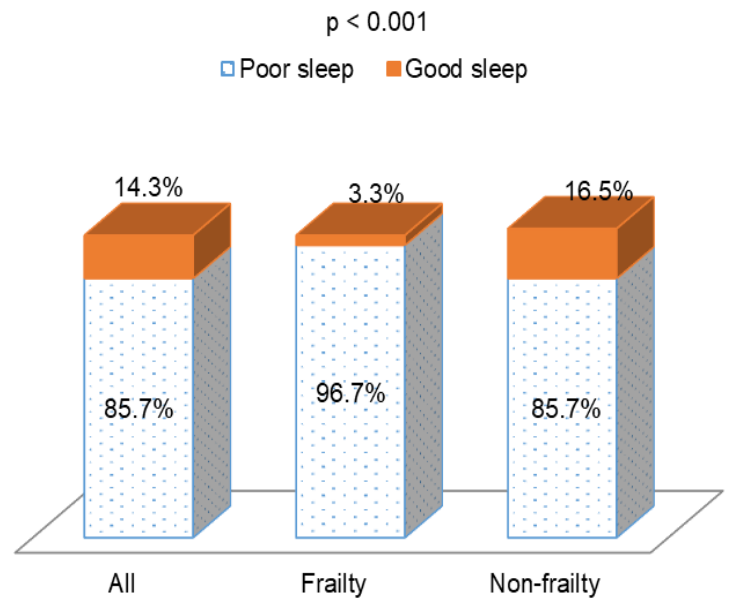

Figure 1 The prevalence sleep disturbance in frailty older adults $(n=903)$

Figure 1 presented the prevalence of sleep disturbance experienced by the participants in the prior month, stratified by frailty status. The majority of the participants $(85.7 \%, 774 / 903)$ reported having poor sleep. Among participants with frailty, 96.7\% (145/150) reported having poor sleep.

\section{PSQI score results}

- Can sleep within 30 minutes

- Cannot sleep within 30 minutes

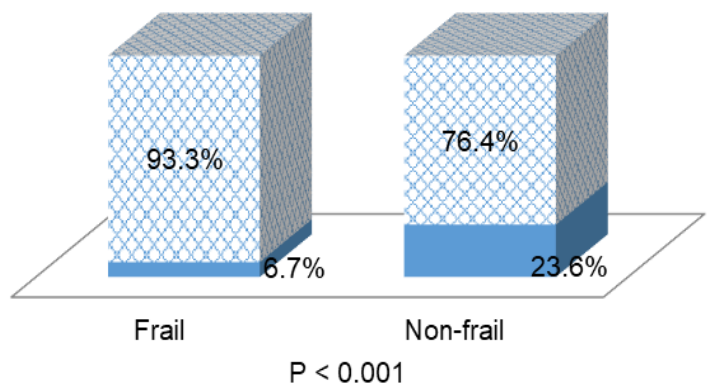

Figure 2. The percentage of sleep latency

$$
\text { ( } n=903 \text { ) }
$$

Almost all frailty participants (93.3\%, $140 / 150$ ) could not fall asleep within 30 , and the majority $(76.4 \%)$ of non-frailty reported having the same problem.

Sleep efficiency was calculated by the following formula:

Sleep efficiency $=$ (total of hours asleep)/ (total of hours in bed) $\times 100$

Only $14.0 \%(21 / 150)$ and $32.0 \%$ of frailty and non-frailty participants had sleep efficiency of more than $85 \%$, respectively. 


\section{$\square$ Sleep efficiency $\geq 85 \% \quad \square$ Sleep efficiency $<85 \%$}

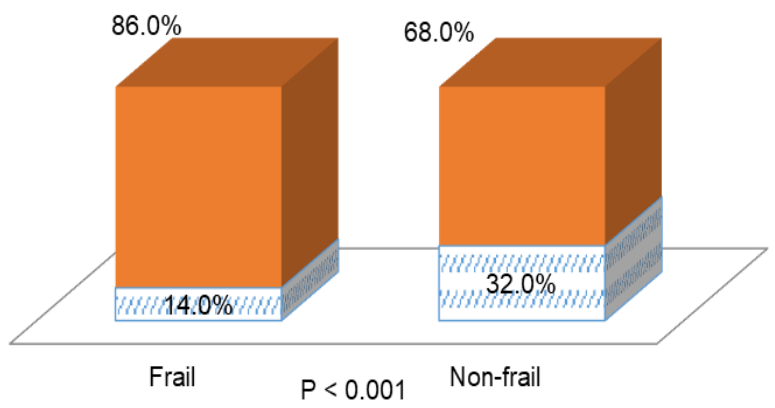

Figure 3. The percentage of sleep efficiency $(n=903)$

During sleep, frailty participants were significantly more likely than non-frailty participants to get up at midnight or early morning, get up to use the bathroom, feel pain, feel hot, cough, have nightmare, and experience difficulty breathing.

Table 2. The characterisitcs of sleep disturbances $(n=903)$

\begin{tabular}{lccr}
\multicolumn{1}{c}{ Classification } & Frailty $(\mathbf{N}=\mathbf{1 5 0})$ & Non- frailty $(\mathbf{N}=\mathbf{7 5 3})$ & $\mathbf{p ~}$ \\
\hline Getting up at midnight or early morning & $143(95.3)$ & $618(82.1)$ & $<0.001$ \\
\hline Getting up to use bathroom & $120(80.0)$ & $505(61.9)$ & $<0.001$ \\
\hline Feeling pain & $118(78.7)$ & $341(45.3)$ & $<0.001$ \\
\hline Coughing & $80(53.3)$ & $348(46.2)$ & $<0.001$ \\
\hline Having nightmare & $76(50.7)$ & $209(27.8)$ & $<0.001$ \\
\hline Feeling difficult to breath & $66(44.0)$ & $277(36.8)$ & $<0.001$ \\
\hline Feeling cold & $34(22.7)$ & $127(16.9)$ & 0.18 \\
\hline Feeling hot & $41(27.3)$ & $130(17.3)$ & 0.01 \\
\hline Other & $12(0.08)$ & $28(0.04)$ & 0.32
\end{tabular}

\section{DISCUSSION}

On average, the frailty participants in our study were older than non-frailty participants $(78.4 \pm 9.0$ vs. $71.8 \pm 8.5)$. This finding is similar to a previous study in Ecuador, in which the average age of the participants was 71 years old $(S D=8)$ and 76 years old $(S D=9)$ in frailty participants. ${ }^{10}$ We observed a higher proportion of women among our participants $(59.5 \%$ compared to $40.5 \%$ for men). Other studies conducted in China and Ecuador similarly had more women than men among their participants. ${ }^{10,11}$ In said studies, the prevalence of frailty was significantly higher among women compared to men, with $69.2 \%$ and $70 \%$ of the frailty participants were women in China and Ecuador, respectively. ${ }^{10,11}$ The prevalence of female in frailty participants slightly increased of the community. This characteristic may be explained by the greater physiological muscle mass loss experienced by women as they age; in addition women are more prone to have sarcopenia, an intrinsic risk for developing frailty syndrome. ${ }^{12}$ 
Most of our participants reported having poor sleep $(85.7 \%)$, and among the frailty participants, almost all (96.7\%) reported having poor sleep. Both observations were higher compared to proportions reported by Sun el al. in China (56.1\%) and Ensrud et al. in the United States (61.0\%), ${ }^{11,13}$ although the sample size in our study was smaller. Almost all frailty participants (93.3\%) could not fall asleep within 30 minutes, while this prevalence in China was just $37.9 \% .^{11}$ Only $14.0 \%$ of our frailty participants had sleep efficiency of more than $85 \%$, much lower than the $43.6 \%$ reported by Sun et al. in China. ${ }^{11}$ The relationship between sleep disturbances and frailty status might be mediated through some mechanisms. Sleep disorders may be a marker of a number of conditions including poor health and comorbidities (cardiovascular disease, depressive symptoms, cognitive dysfunction, and functional disabilities), which by themselves impair sleep and increase the likelihood of greater frailty status. Besides, sleep disturbances and frailty or its components have both been linked to alterations in biochemical pathways including lower endogenous testosterone levels, reduced renal function, and elevations in proinflammatory cytokines. ${ }^{13}$

\section{CONCLUSION}

The prevalence of sleep disturbance among frailty older adult was extremely high in our study. Medical staffs should pay greater attention to sleep quality in frailty patients.

\section{REFERENCES}

1. Vu HTT, Nguyen TX, Nguyen TN, et al. Prevalence of frailty and its associated factors in older hospitalised patients in Vietnam. BMC Geriatr. 2017; 17(1): 216.

2. Kojima G, lliffe S, Jivraj S, Walters K.
Association between frailty and quality of life among community-dwelling older people: a systematic review and meta-analysis. Journal of epidemiology and community health. 2016; 70(7): 716-721.

3. Kojima G, Liljas AEM, lliffe S. Frailty syndrome: implications and challenges for health care policy. Risk Manag Healthc Policy. 2019; 12: 23-30.

4. Xue QL. The frailty syndrome: definition and natural history. Clinics in geriatric medicine. 2011; 27(1): 1-15.

5. Kurina LM, McClintock MK, Chen JH, Waite LJ, Thisted RA, Lauderdale DS. Sleep duration and all-cause mortality: a critical review of measurement and associations. Annals of epidemiology. 2013; 23(6): 361-370.

6. Mannion H, Molloy DW, O'Caoimh R. Sleep Disturbance in Older Patients in the Emergency Department: Prevalence, Predictors and Associated Outcomes. International journal of environmental research and public health. 2019; 16(19).

7. Le Dinh D, Thuong NH. Cognitive impairment and sleeping disorder among the elderly at communities in Hue city, Vietnam. 2017.

8. Van Cauter E, Spiegel K, Tasali E, Leproult R. Metabolic consequences of sleep and sleep loss. Sleep medicine. 2008; 9 Suppl 1(0 1):S2328.

9. Bieniek J, Wilczyński K, Szewieczek J. Fried frailty phenotype assessment components as applied to geriatric inpatients. Clinical interventions in aging. 2016;11: 453-459.

10. Del Brutto OH, Mera RM, Sedler MJ, et al. The Effect of Age in the Association between Frailty and Poor Sleep Quality: A Population-Based Study in Community-Dwellers (The Atahualpa Project). Journal of the American Medical Directors Association. 2016; 17(3): 269-271. 


\section{JOURNAL OF MEDICAL RESEARCH}

11. Sun XH, Ma T, Yao S, et al. Associations

of sleep quality and sleep duration with frailty and pre-frailty in an elderly population Rugao longevity and ageing study. BMC Geriatrics. 2020; 20(1): 9.

12. Espinoza SE FL. Risk factors for frailty in the older adult. Clin Geriatr Med. 2007: 15 - 37. 13. Ensrud KE, Blackwell TL, Redline S, et al. Sleep disturbances and frailty status in older community-dwelling men. Journal of the American Geriatrics Society. 2009; 57(11): 20852093. 